\title{
Increasing the Dynamic Range of a Transient Recorder by Using Two Analog-to-Digital Converters
}

\author{
Ronald C. Beavis \\ Department of Chemistry, New York University and Department of Pharmacology, New York University \\ Medical School, New York, New York, USA
}

\begin{abstract}
This article describes an algorithm for recording transient voltages with enhanced dynamic range by using two 8-bit analog-to-digital converters (ADCs). The method requires each transient to be recorded in both ADCs, with different input voltage gains. The transients then are compared and combined to produce a single signal that has less digitization noise and greater dynamic range than signals recorded by either ADC alone and with no decrease in the sampling rate of the ADCs. The selection of operating parameters is considered and guidelines are established for the performance of this type of transient recorder. The system described here was built as a data acquisition device for a time-of-flight mass spectrometer; however, the algorithm could be applied to other types of ADC-based applications to extend their dynamic range. (J Am Soc Mass Spectrom 1996, 7, 107-113)
\end{abstract}

$\mathrm{T}$ The current resurgence in the use of time-of-flight mass spectrometers has been brought about by new ion sources and the availability of improved data recording electronics. These new ion sources-matrix-assisted laser desorption [1] and electrospray ionization [2] - can produce ions that take advantage of the unlimited high mass range of a timeof-flight mass spectrometer. The use of resolution enhancement methods, such as ion mirror energy correction [3] (reflectrons) and delayed source extraction [4], makes the time-of-flight instrument a versatile device for the biopolymer analysis laboratory.

Even with these new ion sources, time-of-flight mass measurement still would not be practical without the development of high bandwidth digital transient recorders. Early research on time-of-flight mass spectrometers was hampered severely by the lack of such recording devices [5]. Researchers had to photograph analog oscilloscope traces or use box-car integrators to record the output of a time-of-flight instrument, and this resulted in clumsy, difficult-to-interpret data sets. In contrast, digital transient recorders convert the rapidly varying output voltage of a detector into an array of numbers that can be stored and manipulated easily with a computer. For time-of-flight mass spectrometers equipped with matrix-assisted laser desorption ion sources, the requirement for a digital transient

Address reprint requests to Dr. Ronald C. Beavis, The Skirball Institute for Biomolecular Medicine, New York University Medical Center, 540 First Avenue, New York, NY 10016. recorder is that it must be able to record long transients ( $>50 \mu \mathrm{s}$ ) with a sampling interval of 1-5 ns. The transient itself is composed of long regions of slowly varying background levels interspersed with fast transient pulses. Accurate measurement of the positions and amplitudes of these fast transient pulses is the key problem in time-of-flight mass measurement. The pulses of interest in a particular transient may differ in amplitude by more than 100:1, but all of the pulses must be recorded with as much fidelity as possible. The range of pulse amplitudes that must be recorded with high fidelity will be referred to in this article as the "dynamic range" of the measurement.

The commercial digital oscilloscopes and computer card-based transient recorders currently available with a sufficiently high sampling rate to meet the requirements of time-of-flight mass spectrometers all are based on 8-bit flash analog-to-digital converters (ADCs). There are difficulties associated with the use of an 8-bit $A D C$ to record input signals that contain pulses of greatly different amplitudes, that is, signals with a wide dynamic range. As an example, imagine a model 8-bit transient recorder, where an input signal $V(t)$ (which is a function of time, $t$ ) is converted into an integer $N(t)$ via the relationship $N(t)$ is equal to the integer part of $255\left(V / V_{\max }\right)$, for $V(t)>0 \mathrm{~V}$ and $V(t)$ $<V_{\max }$. More careful examination of the limits makes it easy to see that any signal less than $V_{\max }(1 / 255)$ is converted into $N=0$ and any signal greater than $V_{\max }$ is converted into $N=255$. Therefore all information about the true amplitude of any signal $V(t)<$ $V_{\max }(1 / 255)$ or $V(t)>V_{\max }$ is lost. The range of $V(t)$ 
where useful information can be stored by the transient recorder (the dynamic range) is therefore $V_{\max }(1 / 255) \leq V(t) \leq V_{\max }$, or a dynamic range of 255:1 (48 dB).

The voltage measurement resolution of an 8-bit ADC is constant within its dynamic range. The minimum voltage difference $(\Delta V)$ that can be measured can be expressed as $\Delta V=V_{\max } / 255$, or more simply expressed in terms of the recorded integers as $\Delta N=1$. The relative voltage resolution (the ratio of the voltage resolution to the measured voltage) also can be written in terms of $N(t)$ :

$$
\begin{aligned}
\Delta V / V(t) & =\Delta N / N(t)=1 / N(t) \text { for } N(t)>0 \\
& =1 \text { for } N(t)=0
\end{aligned}
$$

Equation 1 implies that the voltage resolution for recording a signal becomes unacceptably coarse when a signal's amplitude is near the lower end of the dynamic range. For example, in the case of an 8-bit ADC, when a signal's amplitude is $V(t)=V_{\max } / 128$, then $\Delta V / V(t)=0.5$, which is clearly too large to obtain an accurate measurement of $V(t)$. If an acceptable relative voltage resolution is defined as 0.1 (a $10 \%$ measurement error), then the useful dynamic range of an 8-bit ADC is from $10 \leq N(t) \leq 255$, or approximately $25: 1$ ( $28 \mathrm{~dB})$.

The high values of $\Delta V / V(t)$ that occur when $N(t)$ $\cong 1$ also affect the signal-to-noise ratio $(S / N)$ [6] in any region of a transient that meets this condition. For example, a direct current (dc) background voltage of $V_{\max } / 255$ with a high frequency noise signal of $\pm 0.1\left(V_{\max } / 255\right)$ will be measured by the ADC as $N(t)=1 \pm 1$. Therefore, the real intensity of the high frequency noise is 10 times smaller than the noise measured by the ADC. Although this is the worst possible case, in the real transients produced by timeof-flight mass spectrometers, the background signals are usually slowly varying small voltages with superimposed high frequency noise, which comes surprisingly close to the conditions in the example. This type of noise can be recognized easily in a transient, because the background will appear to have large "steps," which correspond to changes of a single integer in the ADC output. Small, real pulses that occur in regions where $N(t) \cong 1$ also appear to be very noisy, regardless of the true signal-to-noise value. It should be noted that some fast ADC circuits have an inherent random inaccuracy in the least significant bit (or two), which results in exaggerated noise in any region of the transient where the $N(t) \cong 1$ condition is met.

These considerations of useful dynamic range and $A D C$-induced noise suggest that an 8-bit ADC has a useful dynamic range of about $28 \mathrm{~dB}$ on a single transient record, assuming that a relative voltage resolution of 0.1 is sufficient. In practice, this resolution is too coarse for measurement of the time-of-flight of an ion in a mass spectrometer. Therefore, successive transients are averaged, which results in improved resolu- tion and smooth curves for large signals. For small signals, the background noise caused by the coarseness of the digitization slowly smoothes out as the number of transients that are averaged increases, but distortions to the original signals caused by the measurement coarseness are not necessarily removed; in fact, they may be exaggerated because the same skewed values are recorded over and over again.

This article describes a simple algorithm that allows the output of two 8-bit ADCs to be added together without distortion, which results in a signal that appears to have been taken with a 12-bit ADC. The combined system therefore has a useful dynamic range of approximately 500:1 $(54 \mathrm{~dB})$ when the $\Delta V / V<0.1$ definition is used. The algorithm requires two identical ADCs with input amplifiers, with the amplifier gains set so that one ADC receives signals with 16 times higher amplitude than the other, that is, a gain ratio $\left(r_{g}\right)$ of 16 . The gains must be set so that no part of the lower gain signal exceeds the dynamic range of its associated ADC. A signal is then recorded by the two $A D C s$ and the digitized transients are stored. The transients are then examined point by point. If a point in the transient from the high gain $A D C$ has a value below 255, then that value is stored in a reconstructed transient record, with the same index value as the original point. If the value is 255 , then the value of the corresponding point in the transient from the low gain ADC is taken, multiplied by $r_{\mathrm{g}}$, and stored in the reconstructed transient record. When all of the points have been examined, the reconstructed transient is stored and ready for either immediate use or further summing or averaging.

A computer program that uses this algorithm has been written and used to collect spectra on a linear time-of-flight mass spectrometer with a matrix-assisted laser desorption ion source. The spectra obtained show that the algorithm works on the types of transients generated by a time-of-flight mass spectrometer and produces a significant improvement in the signal-tonoise ratio of small signals.

\section{Experimental}

\section{Dynamic Range Enhancement Algorithm and Data Acquisition System}

The algorithm used is expressed below in structured English format. It must be understood clearly that this algorithm will not work with summed, averaged, or smoothed transients as the input transients (TRANSIENT \#1 and TRANSIENT \#2). If such altered transients were to be used, then none of the conditional statements (IF ..., THEN ...) would be mathematically valid and serious peak distortions would result. These distortions would be difficult to detect by visual inspection, but would seriously skew peaks and make consistent, accurate peak centroid assignments impossible. 


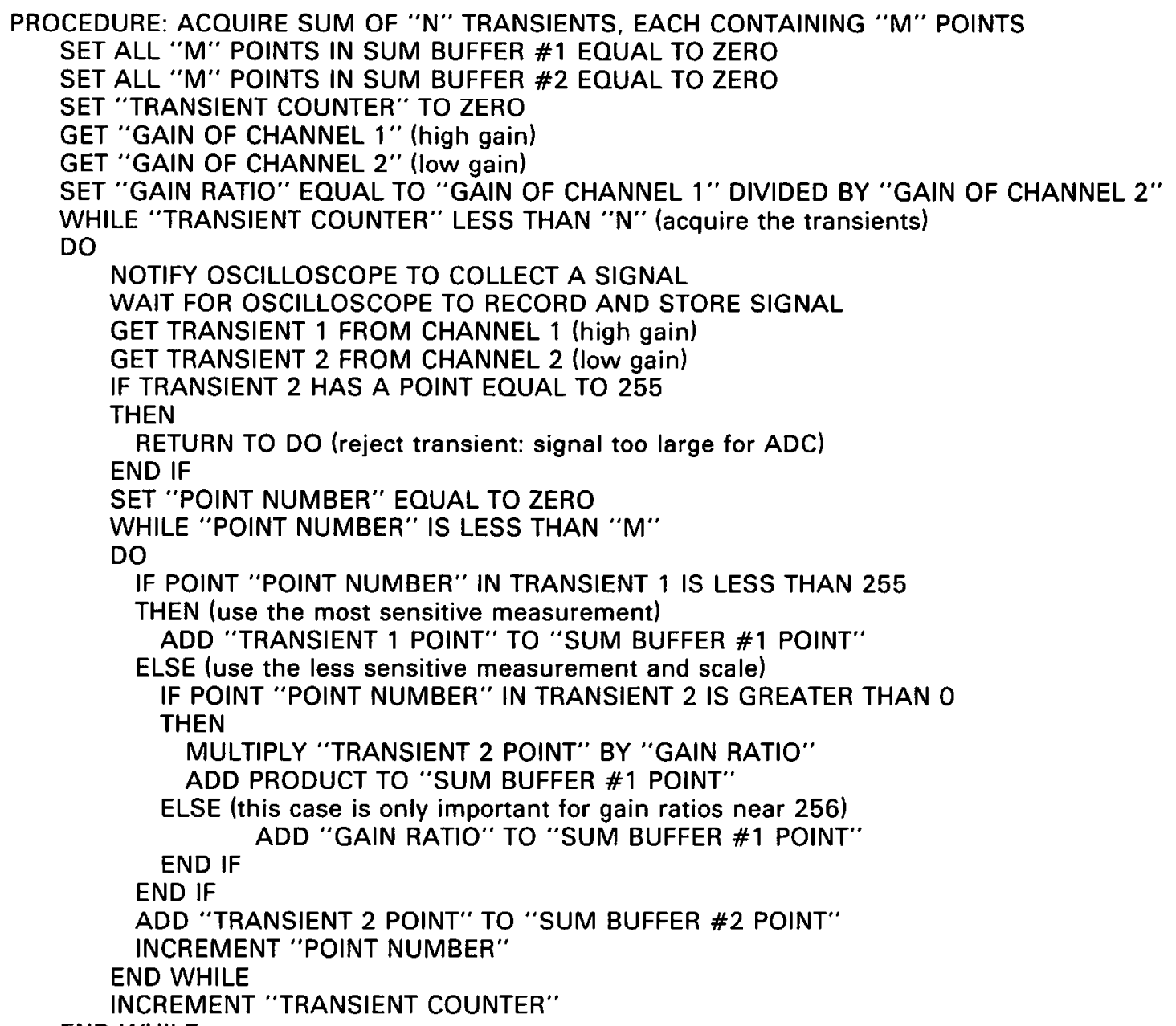

\section{END WHILE} END PROCEDURE:

This algorithm was expressed in $\mathrm{C}++$ and incorporated into a data acquisition program in the author's laboratory. The two ADCs were the two channels of a LeCroy (Chestnut Ridge, NY) 9350M digital oscilloscope $(500-\mathrm{MHz}$ sampling rate with 250,000 points of memory per channel) with the input gains adjusted by means of the oscilloscope's variable gain input amplifiers. The gains used for these experiments were as follows: channel 1 gain $=10 \mathrm{mV} /$ division; channel 2 gain $=160 \mathrm{mV} /$ division $\left(r_{\mathrm{g}}=16.0\right.$; dynamic range $=$ $72 \mathrm{~dB}$ ). See Results and Discussion for a discussion of the required accuracy of $r_{g}$. The signal for the ADCs was conducted from the detector (described in subsequent text) on a $50-\Omega$ coaxial cable, terminated with 50 $\Omega$ at the detector end. The coaxial cable was attached to a tee-connector, with one free end of the connector attached to channel 2 of the oscilloscope directly and the other free end attached to channel 1 with a short (8-cm) stub of $50-\Omega$ cable. The termination impedance on channel 2 was set at $50 \Omega$ and the termination impedance on channel 1 was set at $1 \mathrm{M} \Omega$. Note that if both input termination impedances are set to $50 \Omega$, the cable transmission line will not be terminated properly, which will result in ringing and other types of signal distortion. The arrangement described here al- lows the same signal to be recorded on both channels, with no appreciable attenuation of the input signal.

The transients recorded by the oscilloscope were exported to a Dell (Austin, TX) XPS P90 computer via an IEEE-488 interface (National Instruments, Austin, TX, TNT-GPIB board) where the transient comparison and reconstruction were performed. SUM BUFFER \#1 contained the summed, reconstructed signal with increased dynamic range and SUM BUFFER \#2 contained the summed signal from the low gain ADC only (i.e., the signal normally obtained by using a single ADC system).

The acquired spectra use scaled intensity (SI) as the ordinate. The scaled intensity was calculated by the expressions

$$
\begin{aligned}
& \mathrm{SI}(t)=100 \cdot I(t) /\left(255 n_{\mathrm{t}}\right) \quad(\text { single ADC }) \\
& \mathrm{SI}(t)=100 \cdot I(t) /\left(255 n_{\mathrm{t}} r_{\mathrm{g}}\right) \quad(\text { dual ADC })
\end{aligned}
$$

where $I(t)$ is the value of the summed transient, $n_{t}$ is the total number of individual transients summed, and $r_{\mathrm{g}}$ is the gain ratio (high gain/low gain). Thus, SI varies from 0 to 100 , where a value of 100 implies a signal of $V=V_{\max }$ for every one of the transients recorded in the low gain ADC. Use of SI makes it easier to compare a recorded transient (or average) 
with respect to the dynamic range of the ADC used to record the signals.

\section{Mass Spectrometry}

The mass spectra were obtained by using a custombuilt mass spectrometer constructed at New York University. The instrument was a linear time-of-flight mass spectrometer with a $1-\mathrm{m}$ flight path and a single field acceleration region. The acceleration potential was +40 $\mathrm{kV}$ for all spectra. The flight tube contained a singlewire ion guide, held at $-50 \mathrm{~V} \mathrm{dc}$. The detector used was a 25 -mm-diameter multichannel plate followed by a gridded electron multiplier (both from Hamamatsu, Bridgewater, NJ), configured so that the front face of the channel plate was at ground with respect to the flight tube.

The matrix-assisted laser desorption ion source used a nitrogen laser (LSI 337ND, Boston, MA) as the light source. The laser light was focused onto the end of a $200-\mu \mathrm{m}$-diameter fiber optic (Newport Corp., Fountain Valley, CA) with a $+20-\mathrm{cm}$ focal length lens. The light was transmitted $1.5 \mathrm{~m}$ through the fiber optic. The output end of the fiber optic was imaged onto the matrix deposit in the ion source via a doublet of $+20-\mathrm{cm}$ focal length fused silica lenses.

\section{Results and Discussion}

\section{Effects on Single Transients}

The utility of the foregoing algorithm was demonstrated by matrix-assisted laser desorption analysis of a mixture of two analytes: an unpurified synthetic peptide and horse skeletal muscle cytochrome $c$. The mixture was constructed so that the signal from the peptide was approximately 50 times more intense than the signal from cytochrome $c$. Considering the preceding discussion of dynamic range, that intensity ratio should result in an unacceptable relative voltage resolution for the cytochrome $c$ peaks that use a single 8-bit ADC, when ADC input gain is set so that the peptide peak is also within the dynamic range of the $A D C$. The dual ADC system described previously $\left(r_{\mathrm{g}}=16\right.$; dynamic range $\left.=72 \mathrm{~dB}\right)$ should increase the relative voltage resolution for such signals sufficiently to see a significant improvement in the fidelity and signal-to-noise ratio $(S / N)$ of the cytochrome $c$ signals.

The mass spectra that result from a single transient signal generated from this mixture are represented in Figures 1 and 2. Figure 1 shows the entire mass spectrum recorded, from $m / z=350-14,000$, by using a single ADC (Figure 1a) and dual ADCs (Figure 1b). The region $\mathrm{m} / z>4000$ has been expanded to show clearly the cytochrome $c$ peaks. The peptide peak's scaled intensity shows that its maximum intensity was approximately $80 \%$ of full scale for the transient recorder system (see eqs $2 a$ and $2 b$ ). Figure $1 \mathrm{a}$ demonstrates that the background noise has been distorted by

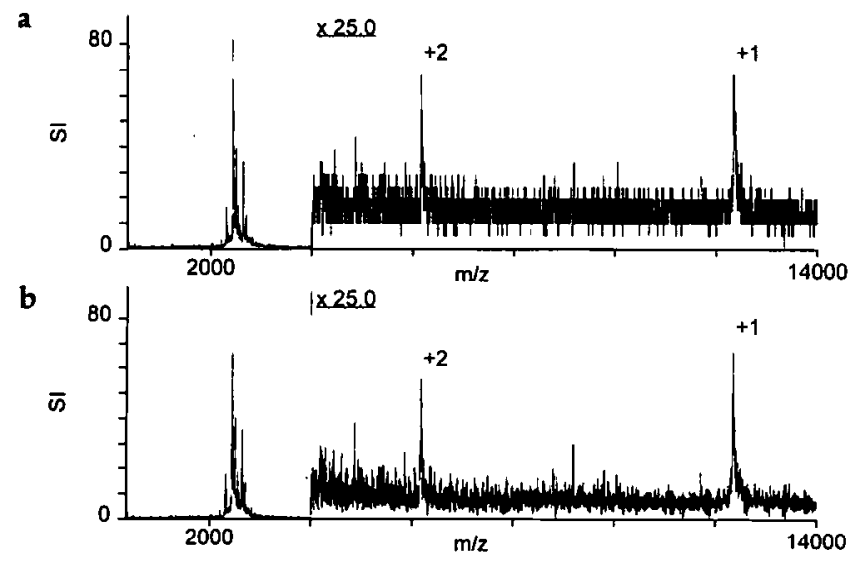

Figure 1. Two spectra constructed from the same single transient. The sample contained an unpurified synthetic peptide mixture and horse skeletal muscle cytochrome $c$ (molar ratio $\cong$ 100:1). The sample was mixed with saturated $\alpha$-cyano-4hydroxycinnamic acid in 2:1, 0.1\% aqueous TFA:acetonitrile, and $0.5 \mu \mathrm{L}$ of the resulting solution was used to form a simple dried droplet deposit. The cytochrome c peaks were labeled " +1 " and " $+2, "$ depending on their charge state. (a) The spectrum obtained from the transient signal by using a single ADC (which corresponds to SUM BUFFER \# 2 in Experimental Section), with the region $m / z>4000$ expanded by 25 times. (b) Spectrum obtained from the same transient signal as (a), but by using the dual ADC system (which corresponds to SUM BUFFER \#1, $r_{\mathrm{g}}=16$ ), with the region $m / z>4000$ expanded by 25 times. SI was calculated using eqs $2 a$ and $2 b$. Every point in the original transient was plotted as recorded, without smoothing or binning.

the ADC's low relative voltage resolution at such low amplitudes, which resulted in the stepped background described previously. Figure $1 \mathrm{~b}$, in contrast, shows almost no background stepping and gives a much more accurate representation of the true background signal. Figure 2 displays the region near the singly charged ion peak for cytochrome $c$. Figure 2 a demonstrates that the stepped character of the background extends to the cytochrome $c$ ion signal, which results in a strongly distorted peak and poor signal-to-noise ratio. Figure $2 \mathrm{~b}$ shows that the dual ADC system effectively can remove this distortion and the voltage resolution-generated noise to give a much clearer record of the real signal.

\section{Effects on Averaged Transients}

The relatively poor voltage resolution obtained on a single transient can be improved by adding transients together to form a summed (or averaged) signal. If the transient signals are not all identical, but the intensity of each pulse varies about some average value, then the averaged signal gradually will approach the average value and the low voltage resolution will become less important as the number of averaged transients increases. This type of averaging scheme is particularly useful for repetitive periodic waveforms, where improvements of several bits in measurement accuracy can be obtained. Averaging also improves the noise produced by either the ADC [when $N(t) \cong 1$ ] or by 


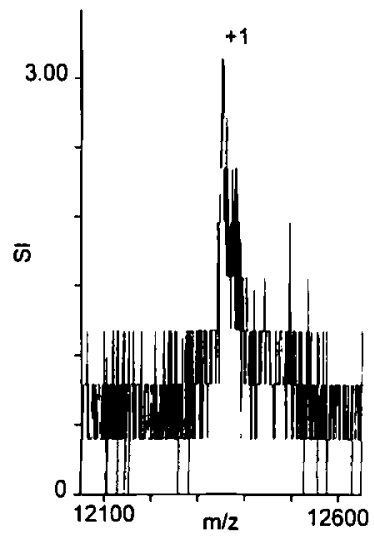

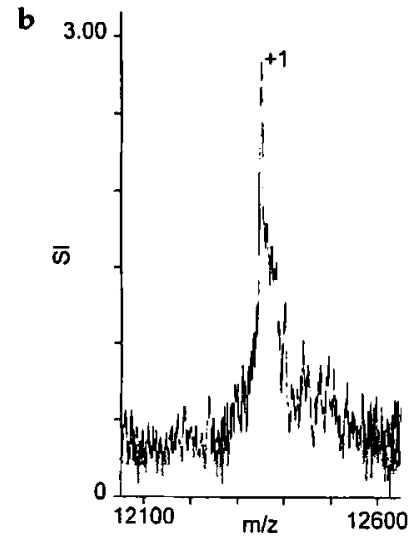

Figure 2. With the same data as in Figure 1a and b, these spectra show the region around the cytochrome $c$ singly charged ion peak. (a) $(S / N=8 \mathrm{~dB})$ and (b) $(S / N=18 \mathrm{~dB})$ correspond to the single $A D C$ and dual $A D C$ systems, respectively. Every point in the original transient was plotted as recorded, without smoothing or binning.

real signals that are uncorrelated with the start of the transient.

The effect of averaging on the two types of noise is different, however. Noise produced by real, uncorrelated signals (such as single-shot noise, thermal noise, inductive pickup, etc.) results in a background that does not increase in intensity as the transients are summed together, because one noise spike is unlikely to be in exactly the same position as another noise spike in a subsequent transient. Therefore, as the number of transients $\left(n_{\mathrm{t}}\right)$ in a summed spectrum increases, the real, correlated signal intensities grow arithmetically while the noise intensity remains the same, which results in a simple expression for the signal-to-noise ratio as a function of $n_{t}$ :

$$
S / N\left(n_{\mathrm{t}}\right) \equiv S / N_{1}+20 \log \left(n_{\mathrm{t}}\right)
$$

where $S / N_{1}$ is the signal-to-noise ratio of any individual transient in the set being averaged, which is assumed to be a constant. The intensity of noise that is produced by an ADC when $N(t) \cong 1$ does not remain the same with averaging. This type of noise behaves according to Poisson statistics, which results in a noise intensity that increases in proportion to $\sqrt{ } n_{\mathrm{t}}$, while the correlated real signals still increase arithmetically, which leads to a different expression for $S / N\left(n_{\mathrm{t}}\right)$ :

$$
S / N\left(n_{\mathrm{t}}\right) \equiv S / N_{1}+10 \log \left(n_{\mathrm{t}}\right)
$$

Clearly, any signal recorded by a real ADC system will have a combination of these two effects. The important point is that the two types of noise have different functional behavior with respect to the signal-to-noise ratio and the noise created by an $A D C$ when $N(t) \cong 1$ is removed less effectively by averaging than normal single-shot noise.

Averaging successive transients works best when the signal amplitude is within the useful dynamic range of the $A D C$. In our example 8-bit $A D C$, any signal below $V_{\max } / 255$ or higher than $V_{\max }$ will result in an ambiguous reading. If the data set used to compute an average transient contains such ambiguous readings, the average will not converge to the true average. These distortions caused by the limited useful dynamic range of an 8-bit ADC are difficult to see in averaged data, although they are easy to recognize in individual transients. For example, if the true average signal is $V_{\max }+1$, with a range of $V_{\max }-1$ to $V_{\max }+$ 2 , the average determined from ADC readings will be $V_{\max }$, rather than the true value.

The relative voltage resolution of an individual measurement also affects the accuracy of an averaged signal. A signal with a true average value of 1.2 $\left(V_{\max } / 255\right)$, which ranges between $1.1\left(V_{\max } / 255\right)$ and $1.3\left(V_{\max } / 255\right)$, will produce an ADC-measured average to $1.0\left(V_{\max } / 255\right)$ exactly, regardless of the number of transients used in the average. Therefore, any pulse in a transient with an average amplitude near the minimum of a transient recorder's dynamic range will be distorted by the coarseness of the ADC's voltage resolution relative to any variability in the pulse's amplitude from transient to transient.

Figures 3 and 4 show the mass spectra obtained by adding together 10 transients by using the same sample and conditions used for Figures 1 and 2. The 10 transients were recorded from successive laser shots and all had similar ion signal intensities. Individual transients were not selected on the basis of their aesthetic character: the results of 10 laser shots in a row

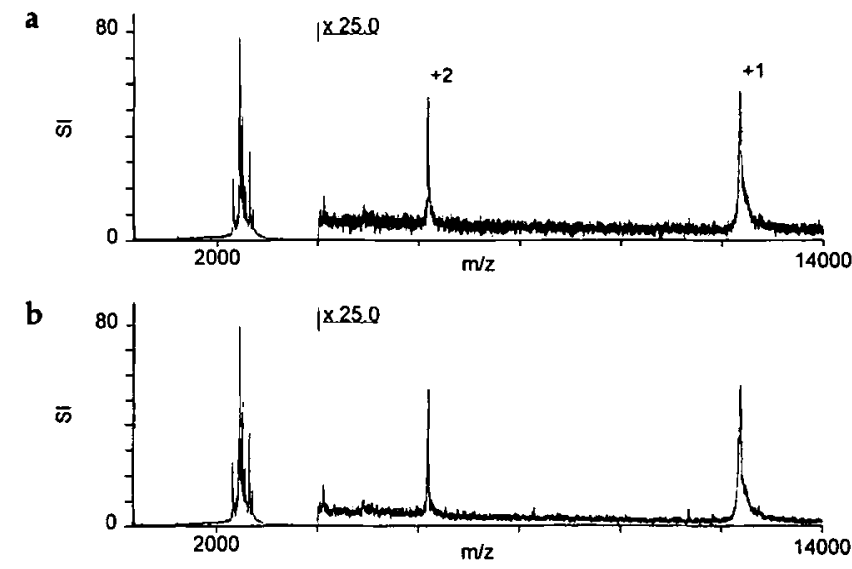

Figure 3. Two spectra constructed from the same set of 10 transient signals. The sample used was the same one used to create Figures 1 and 2 . The most intense peak's signal in each of the individual transients was within $10 \%$ of the average value. The cytochrome $c$ peaks were labeled " +1 " and " +2 ," depending on their charge state. (a) The spectrum obtained from 10 transient signals by using a single ADC (which corresponds to SUM BUFFER \#2 in Experimental Section), with the region $m / z>4000$ expanded by 25 times. (b) Spectrum obtained from the same 10 transient signals as (a), but by using the dual ADC system (which corresponds to SUM BUFFER \#1, $r_{g}=16$ ), with the region $m / z>4000$ expanded by 25 times. Every point in the original transient was plotted as recorded, without smoothing or binning. 

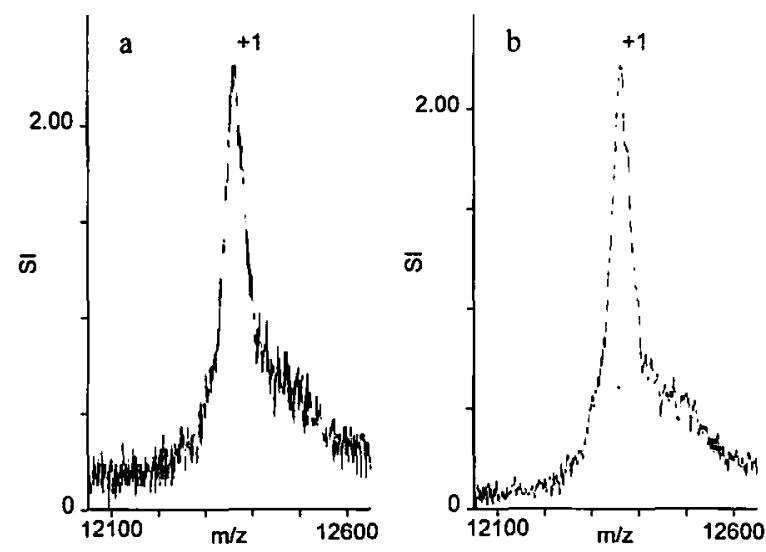

Figure 4. With the same data as in Figures $3 a$ and $3 b$, these spectra show the region around the cytochrome $c$ singly charged ion peak. (a) $(S / N=23 \mathrm{~dB})$ and $(\mathrm{b})(S / N=32 \mathrm{~dB})$ correspond to the single ADC and dual ADC systems, respectively. Every point in the original transient was plotted as recorded, without smoothing or binning.

were used. The scaled intensity shows that the average signal represents approximately $80 \%$ of the dynamic range available. Figure $3 a$ resulted from the single $\mathrm{ADC}$ recording and Figure $3 \mathrm{~b}$ from the dual $\mathrm{ADC}$ system $\left(r_{\mathrm{g}}=16\right)$. The background still shows stepping in Figure 3a, although the effect of averaging 10 transients has reduced the relative size of the steps. The background in Figure $3 \mathrm{~b}$ also has decreased, presumably because the noise in Figure $1 \mathrm{~b}$ was not phasecorrelated with the firing of the desorption laser. The region near the singly charged cytochrome $c$ peak is shown in Figure 4 . The average collected by the single ADC system (Figure 4a) still has significant noise on the signal itself. Figure $4 \mathrm{~b}$ was collected with the dual ADC system $\left(r_{g}=16\right)$. The protein ion peak has a significantly higher signal-to-noise ratio than Figure $4 a$ and the peak has a more clearly defined shape.

\section{Selection of an Appropriate $r_{g}$ Value}

The gain ratio between the input amplifiers on the two ADCs in the dual ADC system is clearly an important parameter that governs the performance and usefulness of the system to extend the dynamic range of a measurement. The following discussion lays out the fundamental considerations involved in the selection of an $r_{\mathrm{g}}$ value for a particular application.

The most important single consideration in selection of an $r_{\mathrm{g}}$ value is the effect that it has on the relative voltage resolution across the dynamic range. Let us consider two extreme cases: $r_{\mathrm{g}}=1$ and $r_{\mathrm{g}}=256$. The first case is trivial: With $r_{\mathrm{g}}=1$ a dual ADC system has no advantage over a single ADC system and should not be used. In the second case (a full 16-bit dynamic range), consider what occurs in the case of signals near the top of the dynamic range of the first (high gain) ADC. A signal that produces an $N_{1}(t)=254$ is recorded as an intensity of 254 with $\Delta V / V=1 / 254$-a precision of $0.4 \%$. A signal that is $1 \%$ larger exceeds the dynamic range of the first ADC and is recorded via the second ADC, which results in a value $N_{2}(t)=0$ and a recorded intensity of 256 , with $\Delta V / V=1-a$ precision of $100 \%$. Such a radical change in the relative precision of a voltage measurement in the middle of the measurement system's dynamic range is not acceptable if signals occur across the entire dynamic range of the device.

A simple criterion to choose the maximum acceptable $r_{\mathrm{g}}$ allowed for a particular measurement system $\left(r_{\text {gmax }}\right)$ is to determine the acceptable voltage resolution when the changeover from the high gain-to-low gain ADC occurs. The relationship between $r_{\text {gmax }}$ and the relative voltage resolution immediately following the changeover from the high gain-to-low gain ADC is given by the expression

$$
\Delta V / V=r_{\text {gmax }} / 256
$$

For the system used to collect the spectra in Figures $1-4$, a value of $r_{\mathrm{gmax}}-16$ leads to $\Delta V / V=0.063$ at the changeover.

Another consideration with regard to $r_{\mathrm{g}}$ is that the amplifiers used must maintain this ratio over the bandwidth used in the measurement. Two-channel digital oscilloscopes provide a good platform for implementation of the dual ADC system because of the excellent reproducibility and high bandwidth of their input amplification gain stages. Any amplifier used in the circuit after the input signal is split and before the ADCs should have specifications similar to an oscilloscope amplifier and the relative gain of the two channels should be measured accurately. The rough estimate of the required stability of $r_{\mathrm{g}}$ during a measurement is that the voltage precision error caused by the variation of $r_{g}$ should be less than the voltage precision error of the low gain ADC measurement.

\section{Conclusions}

Use of a simple algorithm to combine the transients recorded by two analog-to-digital converters with different input gains enabled a new transient to be reconstructed that has a wider dynamic range than either of the individual transients ( $72 \mathrm{~dB}$ compared to $48 \mathrm{~dB}$ for either individual transient). The reconstructed transient can be used to create time-of-flight mass spectra with better signal-to-noise ratios and less small signal distortion than spectra recorded with conventional, single ADC methods.

The system used as an example in this article has a dynamic range of 12-bits and uses two ADCs with an input gain ratio of 16 . There is no fundamental reason why a transient recorder system should be limited to two ADC channels. An oscilloscope with four channels and four independent 8-bit ADCs could be configured to have the same relative voltage resolution characteristics as the two-channel system, but with a dynamic 
range of 20 bits (120 dB). Simple extrapolation of the results presented in this article suggests that a transient recorder with an arbitrary dynamic range can be constructed from a collection of sufficient 8-bit ADCs, input amplification stages, and a computer.

Another application of the same scheme would allow the construction of a composite ADC with a large dynamic. Two 8-bit ADCs with fixed gain input amplifiers (e.g., $r_{\mathrm{g}}=16$ ) could be set up as described here. If the outputs of the ADCs were examined by an embedded controller before being stored in memory, the controller could make the same decisions made by the off-line computer described here, only much faster. The result of the algorithm could then be stored directly in memory without the need to store the individual transients for later comparison. This type of composite ADC could be constructed with an arbitrary number of stages and would result in measuring devices with both a very high dynamic range and speed comparable to a single 8-bit flash ADC.

\section{References}

1. Chait, B. T.; Kent, S. B. H. Science, 1992, 257, 1885.

2. Verentchikov, A. N.; Ens, W. E.; Standing, K. G. Anal. Chem. 1994, 66, 126.

3. Karataev, V. I.; Mamyrin, B. A.; Shmikk, D. V. Sov. Phys.-Tech. Phys. 1972, 16, 1177.

4. Brown, R. S.; Lennon, J. J. Anal. Chem. 1995, 67, 1998.

5. Wiley, W. C.; McLaren, I. H. Rev. Sci. Instrum. 1955, 26, 1150.

6. Hioki, W. Telecommunications; Prentice-Hall: Englewood Cliffs, NJ, 1995; pp 400-401. 